\title{
LE CONTRAT « RETOUR AUX SOURCES » SUR LE BASSIN RHÔNE-MÉDITERRANÉE-CORSE : BILAN ET PERSPECTIVES.
}

\author{
I. LEBEL (1), J.Y. MENELLA (1), M. LE CORRE (2,3)
}

(1) Association Migrateurs Rhône-Méditerranée (MRM), Zl du Port Fluvial, Chemin des Ségonnaux, 13200 Arles, France.

(2) Institut National de la Recherche Agronomique, Laboratoire d'Écologie Aquatique, 65 Rue de Saint-Brieuc, 35042 Rennes Cedex, France.

(3) École Nationale Supérieure Agronomique de Rennes, Département Environnement et Exploitation des Ressources Naturelles, 65 rue de Saint-Brieuc, 35042 Rennes Cedex, France.

\section{RÉSUMÉ}

Sur le bassin Rhône-Méditerranée-Corse (RMC), le Contrat "Retour aux Sources " prévoyait des actions pour l'alose (Alosa fallax) sur l'Aude, sur l'Hérault et sur le Rhône, en aval de la confluence avec l'Ardèche, ainsi que sur le Gardon, son affluent de rive droite le plus aval.

Sur l'Aude et l'Hérault, le Contrat «Retour aux Sources " a été mené par le Conseil Supérieur de la Pêche (CSP). Cinq obstacles ont été ou seront bientôt aménagés pour le passage des poissons migrateurs à la faveur de travaux de réfection ou d'opérations réglementaires qui ont souvent pris plusieurs années avant d'aboutir du fait du manque de mesures financières incitatives. Dans tous les cas, les voies de migration n'ont été rétablies que dans les portions les plus aval de ces cours d'eau.

Sur le Bassin du Rhône, le Plan Migrateurs Rhône-Méditerranée, coordonné par l'association MRM, a été étendu à deux autres affluents de rive droite (Cèze et Ardèche) qui présentaient également de grandes potentialités selon les données historiques disponibles pour l'alose.

Lobjectif le plus amont de ce Plan qui a débuté en 1993, a été atteint puisque depuis 1998, les aloses peuvent remonter dans les Gorges de l'Ardèche grâce aux aménagements réalisés sur le Rhône (adaptation de trois écluses de navigation pour le franchissement des grands ouvrages hydroélectriques par cette espèce) et sur l'Ardèche (modification de deux seuils afin de les rendre franchissables et construction d'une passe à poissons).

En revanche, sur le Gardon et la Cèze, le Plan Migrateurs a pris du retard puisqu'une seule passe à poissons a été réalisée sur les onze prévues. Cependant, une nouvelle passe sera bientôt construite sur le Gardon et trois projets sont actuellement à l'étude. 
Parallèlement aux aménagements, trois types d'études ont été réalisées, à savoir

(1) des études préliminaires aux aménagements (repérage des frayères potentielles, définition du mode de fonctionnement des écluses de navigation), (2) des études de contrôle des résultats des aménagements réalisés, (3) des études scientifiques visant à mieux connaître les populations méditerranéennes d'aloses.

Par ailleurs, des opérations de communication ont été menées en utilisant une exposition itinérante et divers documents (bandes dessinées, recueils de recettes, plaquettes, vidéo).

Les objectifs de cette première partie du Contrat « Retour aux Sources » n'ont pas été totalement atteints, notamment sur le Rhône, comme l'a montré le récent bilan des actions entreprises de 1993 à 1998.

Le prochain Plan Migrateurs sui le bassin RMC, qui sera vraisemblablement étendu à l'anguille, devrait pouvoir être plus évaluable puisque des objectifs plus précis (notamment d'ordre quantitatif), des stratégies d'aménagement et de communication adaptées au contexte socio-politique ainsi qu'une méthodologie de suivi des résultats des aménagements (en particulier, mise en place de stations de comptage et suivi halieutique) sont en cours d'élaboration.

Mots-clés : Alose, Plan Migrateurs, Rhône-Méditerranée-Corse, aménagements, études, passes à poissons.

\section{CONTRACT « BACK TO THE ORIGINS » ON THE RHONE-MEDITERRANEE-CORSICA BASIN : RESULTS AND PROSPECTS.}

\section{ABSTRACT}

On the Rhone-Mediterranée-Corsica basin, the «Back to the Origins " contract anticipated actions for the shad (Alosa fallax) on the rivers Aude, Herault and on the Rhone, dowstream from its confluence with the Ardeche, and also on the Gardon, its first tributary on the right shore.

On the Aude and Herault rivers, the contract was carried out by the Fishing Superior Council (CSP). Five obstacles have been filted, or will be soon, for the passage of migratory fish, and this during repairs or other operations according to regulations, which often took several years to materialize because of a lack of incitative financial actions. In every case, the migratory paths were reinstated only along the lower parts of these rivers.

On the Rhone basin, the Migratory Plan, in coordination with the MRM Association: was extended to two other tributaries, the Ceze and Ardeche rivers, which presented great historical potential for the shad.

The highest upstream objective of the Plan, started in 1993, has been reached as since 1998, the shad can reach the river Ardeche Gorges through developments achieved on the Rhone (adaptation of three navigation locks for the passage of large hydro-electrical works), and, on the river Ardeche (modification of two levels to make them passable, and construction of a fish pass). 
On the other hand, on the rivers Gardon and Ceze, the Migratory Plan is being scheduled : only one fish channel has been made while eleven were forecast. However, a new channel will soon be built on the Gardon and three projects are now under consideration.

In parallel with those developments, three types of studies have been realized :

(1) research prior to developments (finding the potential mating pools, definition of the method of functionment of navigation locks), (2) control of the results of the developments already achieved, (3) scientific research to improve the knowledge of the mediterranean shad population.

Furthermore, operations in communications have been realized using expositions and various documents (comic strips, recipe books, pamphlets, videos).

The objectives of this first part of the « Back to the Origins » contract haven't been totally achieved, especially on the Rhone, as shown by the recent evaluation of the actions carried out from 1993 to 1998.

The next Migratory Plan on the RMC basin, that will be probably extended to the eel, should be more effective because of more precise and quantitative objectives, of better communication strategies, adapted to the socio-political context and also a methodological follow-up of the development results (particularly installation of counting posts and a fishing survey), all these under elaboration.

Key-words : Shad, Migratory Plan, Rhone-Mediterranean-Corsica, developments, research, fish channels.

\section{INTRODUCTION}

Sur le bassin Rhône-Méditerranée-Corse (RMC), le Contrat « Retour aux Sources » prévoyait, sur la période 1991-1995, des actions sur l'Aude, l'Hérault et le bassin du Rhône concernant la restauration des populations d'alose.

Sur l'Aude, trois thèmes de travail avaient été identifiés : (1) la restauration de la libre circulation des poissons avec la construction d'une passe migratoire sur le seuil de Moussoulens, (2) une évaluation du stock lors du contrôle de la migration dans cette passe ef (3) la restauration du milieu en révisant le débit réservé à l'aval de Moussoulens afin de réhabiliter les zones de croissance des alosons.

Sur l'Hérault, les actions suivantes avaient été prévues : (1) la restauration de la libre circulation avec l'aménagement des seuils d'Agde, de Florensac et de Saint-Thibéry, ainsi que (2) l'évaluation du stock par la quantification des passages et l'étude des rythmes de migration par piégeage ou observations vidéo dans les passes migratoires.

Sur le bassin du Rhône, en aval de la confluence avec l'Ardèche, deux axes de travail avaient été définis : (1) la restauration de la libre circulation avec l'aménagement des écluses de navigation des grands aménagements hydro-électriques de Vallabrègues, d'Avignon et de Caderousse ainsi que de sept barrages sur le Gardon (premier affluent de rive droite), et (2) l'évaluation du stock par quantification des passages à l'écluse de Vallabrègues et enquête sur les captures réalisées par la pêcherie. Cependant, sur ce dernier bassin, les objectifs du Contrat «Retour aux Sources " ont été élargis.

En effet, de 1988 à 1991, faisant suite à la demande du Ministère de l'Environnement, la DIREN-Délégation de Bassin RMC et le Service de la Navigation Rhône-Saône, en collaboration avec de nombreux organismes, ont établi le Schéma de 
Vocation Piscicole du Rhône qui proposait : (1) le rétablissement de la circulation de l'alose sur l'axe rhodanien et ses affluents en aval de la confluence avec l'Ardèche, ainsi que la protection des frayères à aloses existantes et potentielles de cette zone ; (2) la restauration de la population d'anguilles jusqu'à Lyon.

Après la validation de ces propositions par le Comité de Bassin en 1991, a été créé l'Atelier Migrateurs dont le rôle était de définir techniquement et financièrement le Plan Migrateurs Rhône-Méditerranée, tant en termes d'études et de communication qu'en matière d'investissements. Cet atelier était constitué globalement des membres du Comité de Pilotage du Schéma de Vocation Piscicole, à savoir les Fédérations de Pêche de l'Ardèche, des Bouches-du-Rhône, de la Drôme, du Gard et du Vaucluse, l'Association des Pêcheurs Professionnels du Bassin Rhône-Saône, la DIREN-Délégation de Bassin $R M C$, le Service de la Navigation Rhône-Saône, le Conseil Supérieur de la Pêche (CSP), l'Agence de l'Eau Rhône-Méditerranée-Corse (RMC), la Compagnie Nationale du Rhôné (CNR) ainsi que des organismes de recherche, le Centre National de Recherche Scientifique (CNRS), et le Centre National du Machinisme Agricole du Génie Rural des Eaux et Forêts (CEMAGREF).

Cet atelier Migrateurs a mené un certain nombre d'études et de travaux el n'a retenu pour la première phase du programme que l'objectif concernant les aloses. Puis ila découpé la zone d'action en deux volets : (1) le volet A comprenant l'axe de navigation du Rhône et ses affluents de rive droite (Gardon, Cèze et Ardèche) ; (2) le volet $B$ constifué par le Bras du Rhône d'Avignon ainsi que par les affluents de rive gauche (Durance Ouvèze et Aygues).

Les travaux du volet $A$ ont été engagés en premier dès 1993, tandis que la définition du volet $B$ devait être précisée après analyse de potentialités de son réseau pour l'alose.

Sur l'Aude et l'Hérault, c'est la Délégation Régionale du Conseil Supérieur de la Pêche de Montpellier qui a conduit les opérations du Contrat «Retour aux Sources".

En revanche, sur le bassin du Rhône, une instance fédératrice a été créée en 1993 à travers l'association Migrateurs Rhône-Méditerranée (M.R.M.). Son rôle est de gérer et de coordonner le Plan Migrateurs Rhône-Méditerranée, tant sur le plan technique que financier, tout en assurant l'information et la sensibilisation du public. Elle regroupait au départ les cinq Fédérations de Pêche participant à l'Atelier Migrateurs, l'Association des Pêcheurs Professionnels du Bassin Rhône-Saône et la Fédération Rhône-Alpes de Protection de la NAture (F.R.A.P.NA). D'autres Fédérations de Pêche, l'Union des Fédérations de Pêche du Bassin du Rhône (U.F.B.R.), l'Association de Défense de l'Environnement de la Vallée du Rhône (A.D.E.V.R.) et la Compagnie Nationale du Rhône ont adhéré ultérieurement.

Pour la réalisation du Plan Migrateurs Rhône-Méditerranée, l'association MRM a bénéficié du soutien technique et financier de nombreuses institutions : le MATE, le CSP. l'Agence de l'Eau RMC, la CNR, Électricité De France (E.D.F.), les Départements des Bouches-du-Rhône, du Vaucluse, de l'Ardèche et du Gard, les Régions Provence-AlpesCôte d'Azur, Rhône-Alpes et Languedoc-Roussillon, l'Institution Interdépartementale des bassins Rhône-Saône (I.R.S.), le Service de la Navigation Rhône-Saône et voies Navigables de France (V.N.F.).

Cet article présente l'état d'avancement de toutes ces actions sur la période 19911998, en s'aidant notamment du bilan des actions entreprises par MRM sur le Rhône (LEBEL et al., 1999). Ce bilan a permis (1) de relier les objectifs de départ aux résultats obtenus en analysant d'une manière critique les études entreprises et les méthodologies qui leur ont été associées, (2) de proposer des solutions d'ordre technique et scientifique pour optimiser le programme de restauration et canaliser les actions prioritaires. 


\section{SITUATION SUR L'AUDE (FIGURE 1)}

L'action majeure prévue dans le cadre du Contrat «Retour aux Sources » sur ce fleuve était la construction de la passe à poissons du seuil de Moussoulens, les deux autres actions ne pouvant être entreprises qu'après sa construction.

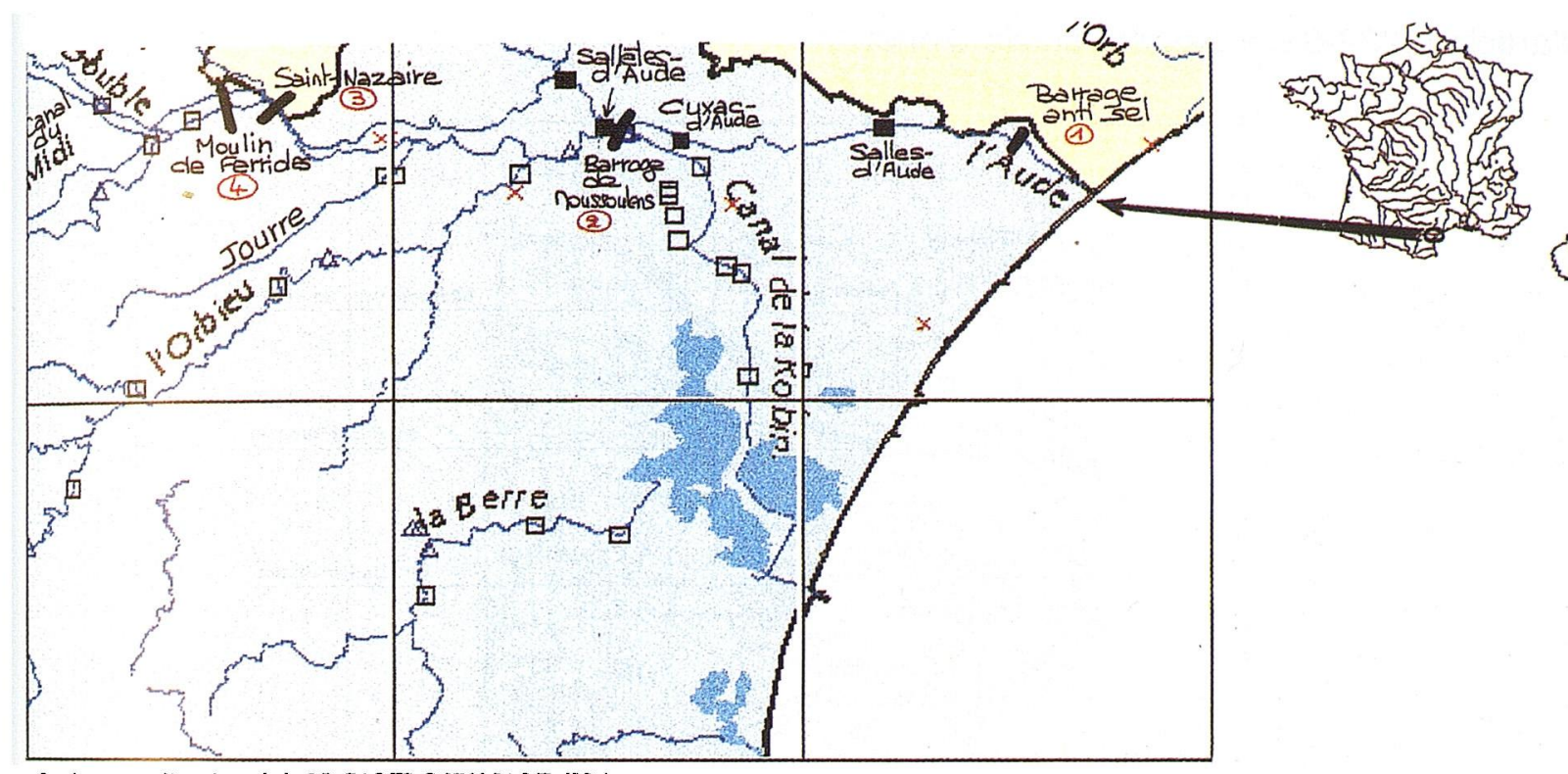

Fond carograshigue issu de la BD CARTO OLON PARS 1994

Figure 1

Localisation des obstacles à la migration sur l'Aude.

Figure 1

Localization of migratory obstacles on the Aude.

En fait, après une longue période d'attente pour se conformer au classement à migrateurs du cours d'eau selon l'article L.232-6, VNF, gestionnaire du seuil, a finalement décidé son aménagement par une passe à poissons après l'intervention du CSP et d'Associations de Protection de la Nature locales auprès de la Préfecture et des Services de l'État.

Ainsi, cette passe à poissons va finalement être construite pendant l'été 1999 et sera donc opérationnelle pour la migration de l'an 2000.

Cependant, en termes de circulation piscicole, des problèmes demeurent sur ce fleuve en raison de l'existence de plusieurs seuils:

- en aval du barrage de Moussoulens, à hauteur du seuil anti-sel de Fleury (seuil à clapets), équipé d'une passe à poissons et sur lequel a été mis en place une gestion particulière des clapets pour permettre la migration des aloses. Ce seuil apparaît toujours difficile à franchir en période de basses ou de hautes eaux;

- en amont du seuil de Moussoulens, où la passe à poissons du seuil de SaintNazaire semble peu efficace pour l'alose. Par ailleurs, plus haut sur le cours d'eau, le seuil de Ferrioles n'est pas encore équipé de passe à poissons. Sa construction a été demandée au propriétaire par les services de l'État après avis du CSP.

D'autre part, sur le plan réglementaire, la modification de la limite de salure des eaux (actuellement en amont du seuil anti-sel) et la protection de la frayère de Moussoulens par arrêté de biotope proposées par le Plan de Gestion 1995-1999 du COmité de GEstion des POissons MIgrateurs (CO.GE.PO.MI.), n'ont pas été réalisées. 


\section{SITUATION SUR L'HÉRAULT (FIGURE 2)}

Sur ce fleuve, parmi les actions prévues dans le cadre du Contrat " Retour aux Sources ", aucune n'a encore été réalisée. Cependant, la construction des passes du seuil d'Agde et du seuil du pont de Florensac sont prévues pour l'été 1999. Par ailleurs, la demande faite par le COGEPOMI d'extension du classement à migrateurs au titre de l'article L.232-6 n'a pas été suivie d'effet.

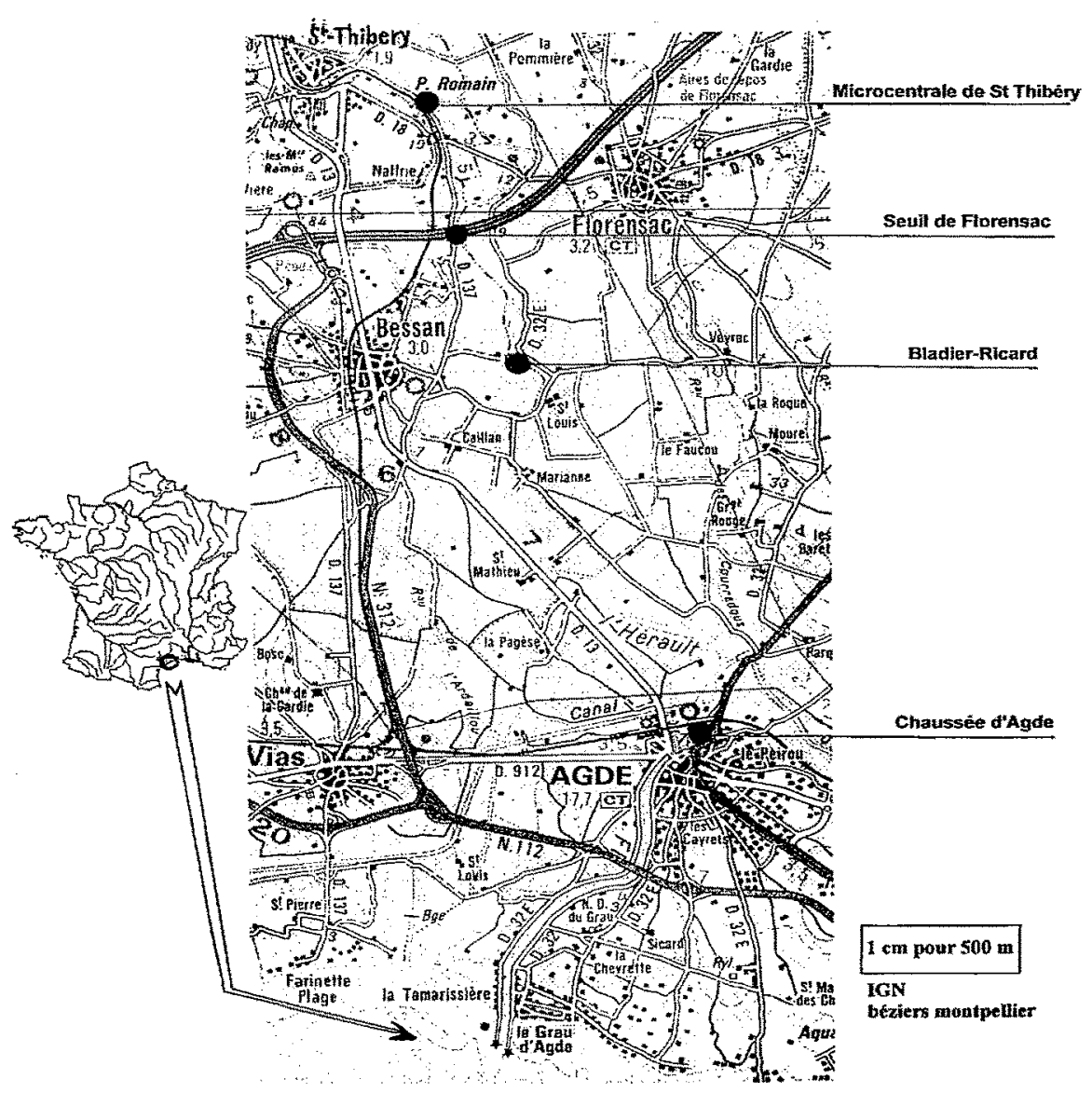

Figure 2

Localisation des obstacles à la migration sur l'Hérault.

Figure 2

Localization of migratory obstacles on the Herault.

\section{SITUATION SUR LE BASSIN DU RHÔNE (FIGURE 3)}

La mise en place du premier volet du Plan Migrateurs Rhône-Méditerranée a nécessité tout un ensemble de travaux d'aménagements et d'ingénierie pour améliorer lia libre circulation des poissons dans la zone concernée. Cet ensemble d'interventions a the accompagné d'études pour suivre ou optimiser les travaux d'aménagement, ainsi que d: mesures réglementaires. 


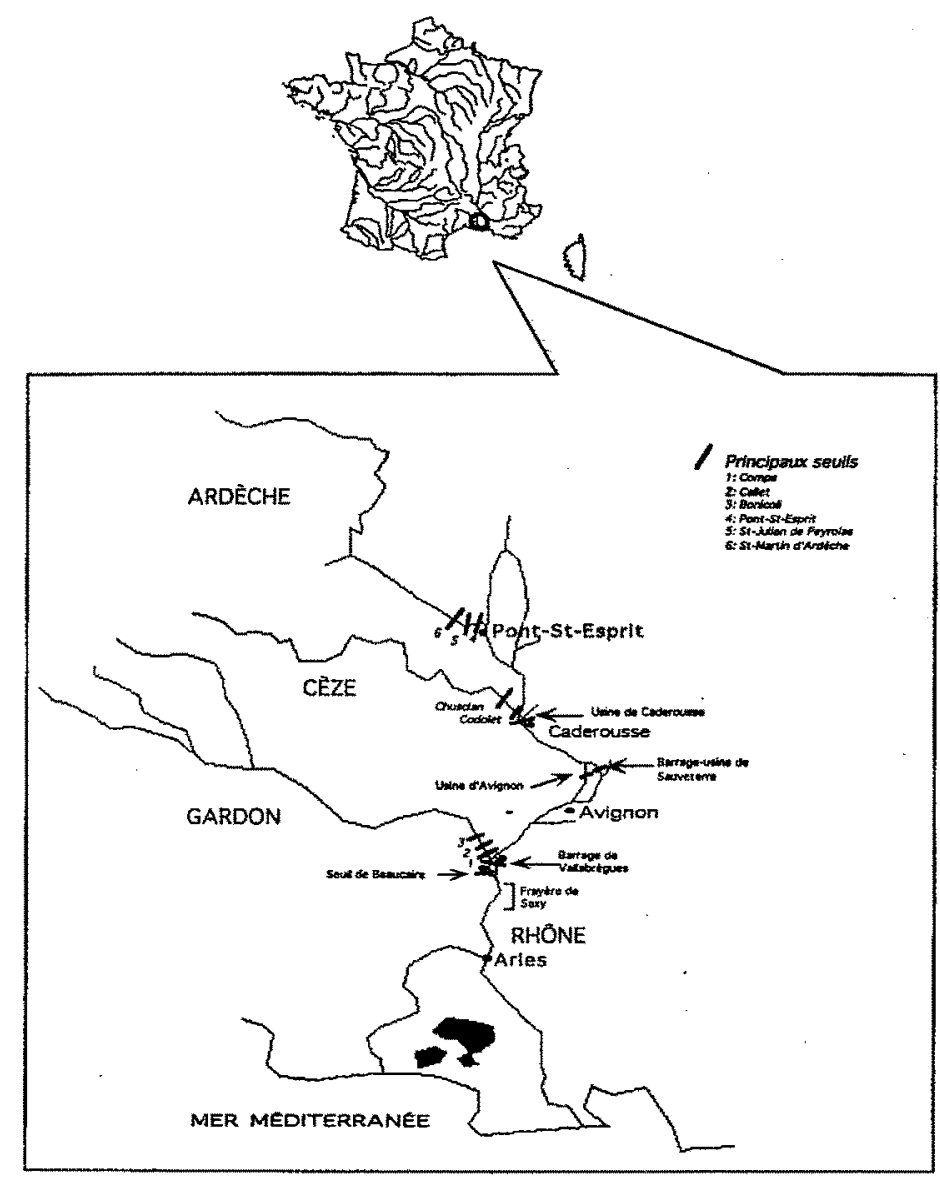

Figure 3

Localisation des obstacles à la migration sur le Rhône et ses affluents.

Figure 3

Localization of migratory obstacles on the Rhone and its tributaries.

\section{Les aménagements}

Les trois écluses de navigation de Vallabrègues, d'Avignon et de Caderousse ont été aménagées pour permettre la réalisation d'éclusées réservées spécialement au passage des poissons. Ceci a consisté à :

- sécuriser les vannes de remplissage du sas, afin de créer un attrait pour que les poissons rentrent dans les écluses ; écluses ;

- motoriser les vantelles, afin de créer un attrait pour que les poissons sortent des

- intégrer dans l'automatisme des écluses les commandes liées aux éclusées à poissons, pour permettre la réalisation de deux à cinq éclusées à poissons par jour entre $8^{\mathrm{H}} 00$ et $18^{\mathrm{H}} 00$, du 15 mars au 30 juin.

Sur l'Ardèche, en aval des Gorges, deux seuils ont été modifiés (seuils de PontSaint-Esprit et de Saint-Julien de Peyrolas) afin de les rendre franchissables et une passe à poissons a été construite sur le troisième (seuil de Saint-Martin d'Ardèche). 
Sur la Cèze, le seuil de Codolet a été équipé d'une passe à poissons et la conception de celle du deuxième seuil (seuil de Chusclan) est en cours.

Enfin, sur le Gardon, aucun des neuf seuils présents (dont le seuil de Beaucaire qui est en fait sur le Rhône court-circuité de Vallabrègues et qui empêche les aloses de se présenter au confluent du Gardon) n'a encore été équipé. Cependant, une passe à poissons sera construite sur deux d'entre eux (Bonicoli et Comps) pendant l'été 1999 el sur le seuil de Beaucaire en l'an 2000.

Dans le cadre du volet A du Plan Migrateurs Rhône-Méditerranée, la migration des aloses est donc de nouveau possible sur le Bas-Rhône jusqu'aux Gorges de l'Ardèche ce qui constitue un progrès très significatif sur ce bassin.

En revanche, des efforts sont encore à faire, notamment sur le Gardon, pour atteindre l'objectif assigné dans le volet A du Plan Migrateurs Rhône-Méditerranée.

\section{Les études}

Trois types d'études obligatoires et/ou complémentaires pour suivre et optimiser les travaux d'aménagement ont été réalisées de 1991 à 1998. Elles ont concerné :

- l'analyse des conditions préliminaires aux aménagements : l'objectif de cette analyse était (1) de faire le bilan des zones colonisées avant le début du programme et des zones potentiellement colonisables notamment en termes de frayères (Bas-Rhône, Gardon, Cèze, Ardèche), et (2) d'ídentifier les obstacles majeurs et les priorités d'aménagement.

- le suivi des aménagements effectués, de façon à mesurer l'efficacité des actions mises en ceuvre en termes de libre circulation, de zones nouvellement colonisées et d'efficacité de reproduction. Ces suivis ont concerné :

(1) le fonctionnement de l'écluse de Beaucaire-Vallabrègues par des techniques de comptage visuel (1991 à 1997) et acoustique (1996 à 1998) suite à l'instauration d’éclusées spécifiques pour le franchissement des aloses ;

(2) le niveau d'occupation - voire de fonctionnalité - des zones de frayères ouvertes sur le Rhône et ses affluents rive droite (menée sur 4 ans de 1995 à 1998) ;

(3) la dévalaison des alosons essentiellement en amont du barrage de Vallabrègues;

(4) l'activité des pêcheries d'aloses, comprenant celle des pêcheries professionnelles et amateurs aux carrelets, localisées en aval du barrage de Vallabrègues et celle de la pêche sportive située aussi bien en aval qu'en amont de cet obstacle (menée de 1996 à 1998), dans le but d'améliorer les connaissances sur la migration et d'obtenir des indices d'abondance du stock.

- l'acquisition des connaissances sur les caractéristiques écobiologiques el taxinomiques de l'alose feinte du Rhône : ce programme d'études scientifiques paraissalit indispensable pour compléter et optimiser les mesures d'ordre administratif et technique; de plus, les connaissances sur les espèces d'aloses présentes dans le Rhône restaientà la fois anciennes et sommaires et devaient donc être approfondies et actualisées ; par ailleurs, la présence ou non de plusieurs espèces ainsi que le statut du taxon rhodanien restaient incertains et demandaient à être éclaircis. 
Ces études ont fait l'objet d'un bilan critique. L'analyse de leurs résultats (LEBEL et al., 1999) a permis :

(1) d'améliorer les connaissances écobiologiques de la population d'aloses du Rhône

Les aloses sont en fait représentées par un seul taxon à savoir l'alose feinte du Rhône (Alosa fallax rhodanensis) (ROULE, 1924). Aucune grande alose ni individu hybride n'ont été observés. L'alose du Rhône présente des particularités spécifiques : elle semble rattachée à l'alose feinte par ses caractères méristiques et son itéroparité, mais se rapproche de la grande alose par ses caractères biométriques (taille, poids, forte croissance) et la longueur de ses parcours de migration (avant les aménagements).

Lidentification génétique confirme l'appartenance au taxon alose feinte mais suggère une possible différenciation de celui de l'Atlantique nord, tout en restant proche de l'alose feinte marocaine (LE CORRE et ALEXANDRINO, données non publiées). Le taxon rhodanien s'est hybridé avec la grande alose (Alosa alosa) introduite en 1953 sur I'Ardèche par HOESTLAND (1958), en faisant éclore 500000 œufs fécondés artificiellement, mais ce phénomène a quasiment disparu. II ne subsiste que sous forme de traces dans le génome de certains individus.

Compte tenu de l'existence d'une seule population, vraisemblablement répartie sur une aire restreinte, cette espèce peut être considérée comme endémique et à statut patrimonial. Cet élément renforce l'intérêt d'un programme de restauration des stocks dans un premier temps au niveau du Rhône et dans un second temps au niveau du bassin méditerranéen français. Ce statut de l'espèce justifie pleinement son inscription dans les programmes d'études concernant la connaissance et le maintien de la biodiversité mis en place récemment en Europe (programme Biodiversitas) et à l'échelon national (programme d'action pour la diversité biologique de la flore et de la faune sauvages). II s'avère par ailleurs nécessaire de connaître exactement l'aire de distribution de ce taxon. Des études seront à mener sur les autres fleuves côtiers méditerranéens, jusqu'en Italie et en Espagne.

Cependant cette étude écobiologique et taxinomique a été réalisée sur des échantillons capturés en aval du barrage de Vallabrègues. Il paraît nécessaire de poursuivre cette approche en amont du barrage afin de savoir si les caractéristiques des poissons franchissant cet obstacle sont identiques à celles des poissons restés en aval ou au contraire si le barrage joue un rôle de filtre structurant artificiellement le flux en différentes composantes biologiques. Ceci est important en termes de structure démographique et de potentiel reproducteur (rapport des sexes, nombre et taille des femelles), et donc de succès pour la restauration des zones amont.

\section{(2) de caractériser la fenêtre spatio-temporelle de migration}

Cette caractérisation a été établie à l'aide de la pêcherie durant trois ans (19961998), en identifiant la période de migration et les facteurs influençant la remontée de reproduction des adultes et en montrant notamment l'impact négatif du barrage (retard important). Cette étude a alors permis de préciser la fenêtre optimale de migration, qui reste assez semblable d'une saison à l'autre quelle que soit l'année (mi-avril à mi-mai). La détermination de cette fenêtre est un élément important à prendre en compte pour optimiser les mesures de franchissement (intensification des éclusées à poissons et des comptages au moment du pic de migration).

\section{(3) de disposer des premiers indices d'évaluation du stock}

Le suivi de la pêcherie d'aloses a permis, à l'aide de l'évaluation des captures et des prises par unité d'effort, de fournir des indices d'abondance relative du stock. 
Cependant, l'on ne dispose pas actuellement de mesures fiables permettant d'estimer le stock. La constitution d'un indice d'abondance à partir des CPUE de la pêcherie aux carrelets est en partie biaisée par sa position sur le bras court-circuité, très proche du barrage (poissons pouvant passer plusieurs fois dans la pêcherie). Par ailleurs, la diminution des captures de la pêche aux carrelets semble plutôt indiquer une réduction de la fraction du stock exploitable par la pêcherie. Cette diminution semble due pour partie à des variations annuelles d'abondance (facteur de 1 à 100) et de répartition spatiale dans le bassin (exemple de la migration 1995 probablement limitée par la forte hydraulicité du Rhône), et pour partie aux mesures mises en place pour augmenter le passage des aloses à Vallabrègues.

De même, la recherche d'un indice d'abondance déduit de la fréquentation des frayères par les géniteurs n'a pas abouti. En effet, le suivi de la frayère de Saxy (portion du Rhône située en aval du premier aménagement, entre Arles et Tarascon, ayant assuréle maintien de l'alose) n'a pas permis de valider la méthode reliant le nombre des bulls à celui des géniteurs. Actuellement la seule mesure d'abondance dont on dispose est le nombre d'individus franchissant le barrage de Vallabrègues. Cependant, les résultais obtenus à partir de la méthodologie utilisée (approche bayesienne avec utilisation d'un modèle exponentiel) restent des indications préliminaires qui demandent à être précisées en augmentant les comptages visuels d'aloses aux périodes de forte migration.

En tout état de cause, au vu de l'ensemble des indices, il semble que le stock ne soit pas résiduel dans la mesure où il supporte une exploitation qui se maintient entre 9 et 12 tonnes par an (captures estimées à partir de carnets de pêche et d'enquêtes de terrain).

(4) de faire le bilan sur lutilisation des éclusées à poissons pour le franchissement du barrage de Vallabrègues

Le fonctionnement de l'écluse en tant que passe à poissons avait été envisagé dès la construction de l'ouvrage de Beaucaire-Vallabrègues. Les paramètres de l'éclusée à poissons ont été progressivement modifiés en fonction des résultats et des contraintes techniques qu'impose la navigation. Ces paramètres ont été testés au cours des campagnes d'études successives pour parvenir à une éclusée type dite optimale, réalisée à raison de une à deux au minimum par jour.

Les résultats des campagnes de comptages visuels et d'échosondage ont montré l'efficacité globale des éclusées à poissons en tant que système de franchissement de l'ouvrage pour le poisson. Cependant, le comptage visuel semble être la technique la plus efficace pour déterminer les espèces et évaluer le nombre des poissons franchissant l'obstacle. Toutefois, cette technique, pour devenir fonctionnelle, doit être améliorée. Elle nécessite le recueil d'un plus grand nombre de données et donc l'augmentation du nombre d'éclusées spécifiques à aloses avec comptage visuel. Par ailleurs, cette récolte des données doit être optimisée durant la période d'intensité maximale de migration. Ced devrait alors permettre de choisir le meilleur modèle d'exploitation des données tout en tenant compte de certaines caractéristiques de la migration des aloses flux de remontée structuré par vagues, passage des obstacles par pics constitués d'un grand nombre d'individus). II faut cependant signaler que l'utilisation et la fiabilité de cette méthode de comptage reste assujettie à un certain nombre de contraintes techniques (manœuvres des portes, intégration dans le calendrier de la navigation fluviale, disponibilité en personnel pour le suivi et les manceuvres), et à des facteurs d'imprécision inhérents à la technique (modalités de comptage sur la largeur totale de l'écluse, variabilité dans la constitution de l'équipe de comptage et dans le seuil de vigilance des opérateurs, fiabilizé des critères de distinction des aloses et des mulets, présence éventuelle d'autres espèces). 
En revanche, la technique acoustique doit être abandonnée, en raison de (1) l'impossibilité de distinguer les espèces franchissant l'écluse et en particulier les aloses des mulets, (2) la faible surface échantillonnée par le dispositif (40\% du volume total en eau), (3) l'impossibilité d'utiliser cette technique lors des éclusées à bateaux pour lesquelles aucun renseignement concernant le transit des poissons n'est connu (GUILLARD et al., 1998), même si des études réalisées sur d'autres cours d'eau ont montré que les passages d'aloses par l'écluse en présence de bateaux sont négligeables en raison de l'absence de phase d'attrait (KIPPER et MILEIKOV, 1968).

\section{(5) d'analyser l'évolution de la zone colonisée}

$\mathrm{Au}$ bout de sept années, l'aire de colonisation des aloses a incontestablement augmenté puisqu'il y avait très peu d'aloses en amont de Vallabrègues (RAMEYE et al., 1976). Ceci est confirmé par (Figure 4):

- une augmentation significative des captures à la ligne en amont du barrage de Vallabrègues, notamment en aval de l'aménagement d'Avignon qui reste avec l'aval de Vallabrègues le site où le plus grand nombre de prises d'aloses est effectué ; par ailleurs, des captures ont été signalées jusqu'à 163 kilomètres de l'embouchure, à Châteauneuf du Rhône et dans l'Ardèche ;

- l'existence de quelques frayères fonctionnelles en amont de l'ouvrage de Vallabrègues (site des Broutières sur l'Ardèche, frayères de Grandbois, de Coudoulas et de St-Montan sur le Rhône court-circuité de Donzère-Mondragon) ;

- la présence d'alosons dans les puits de turbine au niveau de Sauveterre et de Caderousse, en provenance des parties amont ;

- l'observation en juillet-août d'aloses adultes bloquées en phase de dévalaison au niveau des turbines des différentes usines, comme à Caderousse en 1993 et 1994.

Cependant, si l'on peut parler d'une extension de la zone colonisée, la surface à nouveau fréquentée et le niveau d'abondance sur le bassin du Rhône et ses affluents restent impossibles à quantifier d'une manière précise compte tenu du caractère très qualitatif des données récoltées et de la manière dont les aménagements ont été programmés dans le temps (Tableau I).

(6) de tester les éléments pouvant être apportés par le suivi de la reproduction des aloses dans l'espace et dans le temps

Lobservation directe d'une activité de reproduction (bulls) reste un indice de colonisation d'un site par les aloses. Elle permet de conclure à l'efficacité des aménagements réalisés en aval de la zone de reproduction. Cependant, l'utilisation de cette méthode paraît limitée pour plusieurs raisons :

- la difficulté de localiser la zone de frai fréquentée par les géniteurs; en effet, cette dernière peut varier dans l'espace et dans le temps au cours d'une même année en raison des fortes variations journalières de débit qui modifient leur typologie et donc le choix de l'emplacement des géniteurs; enfin, la répartition spatiale des géniteurs en période de reproduction est en partie liée aux conditions hydrologiques de migration sur le bassin (indépendamment des dispositifs de libre circulation) et aux caractéristiques annuelles du stock (abondance, structure d'âge et de taille) ;

- l'absence de relation formalisée entre le nombre de bulls et la production de juvéniles d'aloses, qui empêche d'évaluer le niveau de fonctionnalité des frayères. 


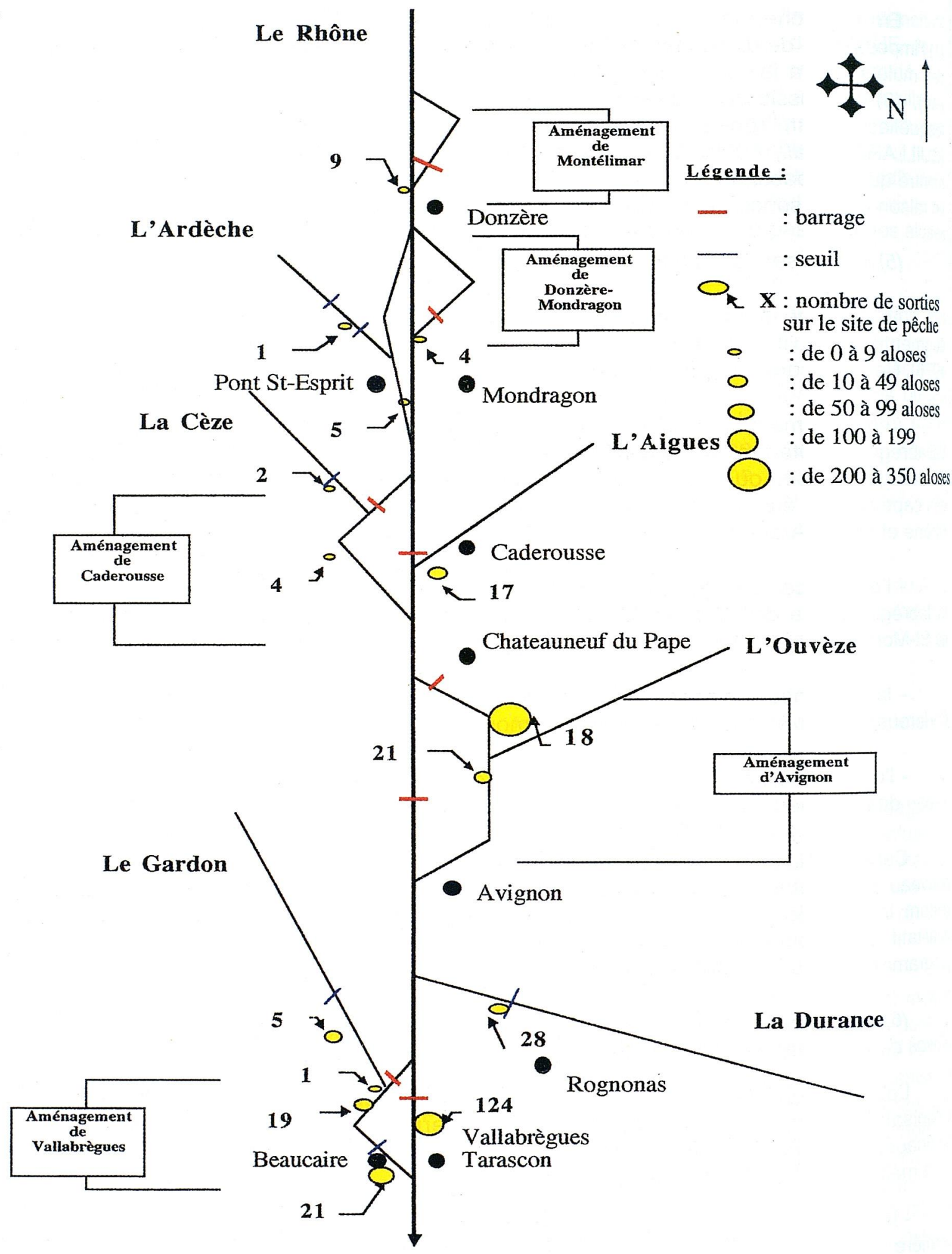

Figure 4

Répartition géographique des aloses capturées à la ligne en 1998 (LEBEL ef ah, 1999). 


\section{Tableau I}

Points de blocage subsistant sur le parcours des aloses (Rhône et affluents de rive droite).

Table I

Blocking points remaining on the shads' course (Rhône and its right bank tributaries).

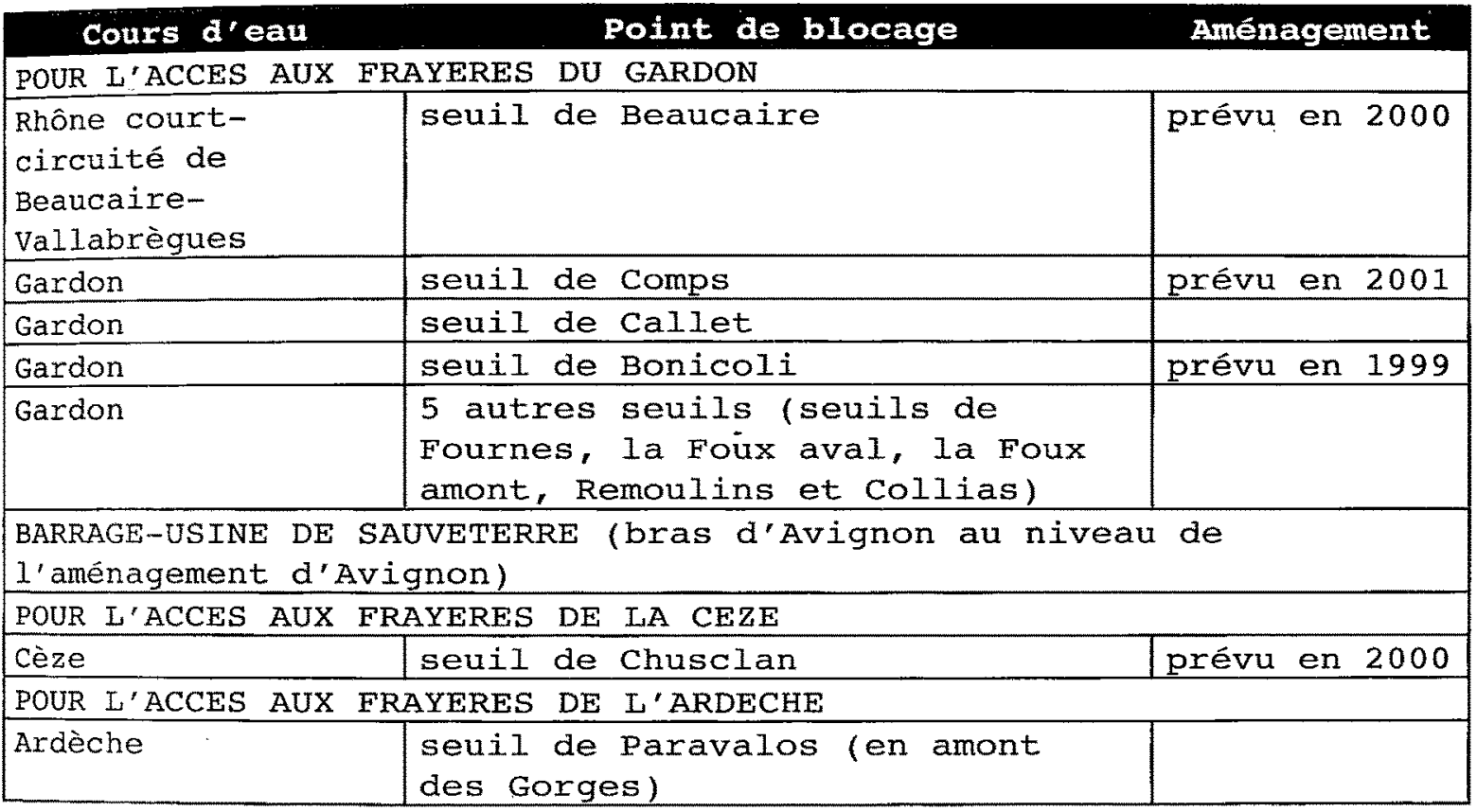

En dépit de ces inconvénients majeurs, si le suivi de la reproduction et la méthode de complage des bulls sur le Rhône lui-même et ses affluents de grande taille s'avèrent être des óvaluations simplement qualitatives, il n'en est pas de même sur des cours d'eau de plus petite taille où il est alors possible à l'aide d'une méthode de suivi simplifiée dans l'espace et dans le temps (visite en période maximale de reproduction sur des frayères préalablement qualifiées de potentielles) de mettre au point un indice de fonctionnalité des frayères.

Par ailleurs, les études effectuées sur la reproduction n'ont pas permis de caractériser la typologie des frayères d'aloses sur le bassin, la reconnaissance de frayères potentielles se faisant toujours à partir de celle établie pour la grande alose sur des fleuves atlantiques de plus petite taille.

\section{Les mesures réglementaires}

Lexension du classement au titre des cours d'eau à migrateurs selon l'article L.232-6 sur le Rhône et ses affluents de la rive droite ainsi que l'arrêté de biotope visant la protection de la zone de frayère de Saxy demandés par le Plan de Gestion 1995-1999 du CO.GE.PO.MI., sont en cours d'instruction.

\section{Les opérations de communication}

Des opérations de communication ont été menées en utilisant une exposition itinérante et divers documents (bandes dessinées, recueils de recettes, plaquettes, vidéo). 
Si ces outils de communication ont permis de mieux faire connaître le Plan Migrateurs dans le tissu associatif du bassin du Rhône, ils n'ont pas permis d'inciter les syndicats intercommunaux gérant les affluents à s'approprier le projet et à s'y impliquer d'une façon plus importante. Ce demi-échec est essentiellement dû à un manque de moyens mais aussi de stratégie de communication.

\section{CONCLUSION ET PERSPECTIVES}

Le bilan du Contrat "Retour aux Sources" est contrasté. En effet, les aménagements réalisés ont permis une amélioration notable de la circulation des aloses, notamment sur le bassin du Rhône, mais le CSP et l'association MRM ont rencontré beaucoup de difficultés pour lancer des programmes d'aménagements sur le Gardon et les fleuves côtiers.

Sur l'Aude, il reste à prendre des mesures réglementaires el à entreprendre de nombreuses actions concernant la protection des milieux, la circulation des poissons ef l'évaluation du stock d'aloses.

Sur l'Hérault, il reste à prendre des mesures réglementaires, à équiper au minimum le seull de St-Thibéry, dernier verrou avant l'accès aux premières frayères convenables pour les aloses, et à évaluer le stock d'aloses.

Sur le Rhône, les objectifs du volet A du Plan Migrateurs Rhône-Méditerranée n'ont pas été totalement atteints, comme l'a montrë le récent bilan des actions entreprises de 1993 à 1998. Ceci est en partie lié à leur caractère prospectấf mais elles ont cependant eu le mérite de constituer une démarche cohérente de travaux d'ingénierie et d'études scientifiques. Par ailleurs, l'équipement du seul axe de navigation sur le Rhône est insuffisant dans la mesure où des zones de blocages persistent en aval du barrage-usine de Sauveterre mais aussi dans les bras court-circuités du Rhône (lorsque le débit est élevé, comme en 1995 et 1999).

De même, s'il a été possible de mesurer qualitativement l'amélioration de la migration de lalose sur le bassin du Rhône grâce aux suivis de la pêche à la ligne et de quelques frayères, il est encore difficile d'apprécier quantitativement la réussite du Plan Migrateurs. En effet, on ne dispose actuellement d'aucune estimation fiable du niveall d'abondance du stock et donc de son évolution depuis la mise en ceuvre du projet.

Ainsi, des améliorations doivent être apportées dans la conduite du projet, surtout si celui-ci est étendu à d'autres espèces de poissons migrateurs, notamment l'anguille, età d'autres zones géographiques.

Ainsi, sur le plan du suivi des résultats, un certain nombre d'actions ou d'études prioritaires, qui s'inscrivent forcément dans le long terme, devront êtres engagées, à savoir :

(1) mettre en place des systèmes fiables et pérennes de suivi de la migration all niveau du premier obstacle afin d'améliorer l'évaluation du stock ou du flux migratoire;

(2) mesurer l'extension de la zone colonisée en multipliant notamment les enquêtes halieutiques sur la peche sportive, qui semblent constituer le moyen d'investigation le plus rapide et le moins coûteux à mettre en ceuvre;

(3) optimiser les mesures mises en oeuvre en harmonisant les méthodes de suivi et en définissant des priorites, compte tenu de la limitation des moyens materiels et humains 
De plus, une nouvelle stratégie doit être menée comme l'a montré l'analyse stratégique du Plan Migrateurs Rhône-méditerranée en cours d'achèvement (ANONYME, 1999) :

(1) après une première phase expérimentale, il faut passer à un projet plus construit en termes d'objectifs, de structures et de moyens;

(2) il faut mieux intégrer le Plan Migrateurs aux projets et aspirations locales pour que les acteurs s'investissent, afin de lever les blocages rencontrés dans le programme d'aménagements;

(3) afin d'améliorer la lisibilité institutionnelle du Plan-Migrateurs, il faut définir un maître d'ouvrage, mandaté par les décideurs, qui assure le cadrage contractuel et le contrôle des conditions de réalisation du projet.

Ainsi, l'association MRM et ses partenaires institutionnels travaillent actuellement sur trois axes :

(1) un volet "Certification Migrateurs ". en créant un label de qualité patrimoniale des rivières et milieux aquatiques, adaptés aux fleuves côtiers et aux affluents du Rhône, qui permettra une meilleure appropriation du Plan Migrateurs par les acteurs locaux;

(2) un volet « Décloisonnement du Rhône », porté par le SDAGE, le Plan d'Action Rhône, le projet de réhabilitation hydraulique et écologique du Rhône, etc. ;

(3) un volet scientifique et technique, support des deux autres pour le développement des connaissances et savoir-faire utiles.

La première étape du Plan Migrateurs concernait l'alose du Rhône.

La seconde a d'ores et déjà démarré et traite des autres fleuves du bassin RMC (Argens, Gapeau et Tavignano).

Enfin, la troisième phase du programme concerne les autres espèces de poissons migrateurs, et en premier lieu l'anguille, dont la situation est préoccupante sur le Bassin RMC.

En effet, dans les parties les plus basses des cours d'eau du bassin, ses populations semblent diminuer depuis plusieurs années. Par ailleurs, les barrages limitent considérablement son aire de répartition, notamment sur le Rhône, alors que le programme actuel d'aménagement des écluses de navigation n'est pas adapté à l'anguille.

Ainsi, dans le cadre du Plan de Gestion 1995-1999 du COmité de GEstion des POissons Mlgrateurs (CO.GE.PO.MI.), deux types d'actions ont été prévus:

- la prise de mesures réglementaires (modification ou définition de la limite de salure des eaux, arrêtés de biotope et classements complémentaires au titre de cours d'eau à migrateurs (article L. 232-6 du Code Rural);

- la réalisation d'études préliminaires sur l'anguille, financées par le MATE et Electricité De France (EDF) :

- une étude bibliographique sur les connaissances actuelles, tant sur le plan biologique que sur le plan exploitation sur le bassin méditerranéen (réalisée par la Station Biologique de la Tour du Valat); 
- une enquête socio-économique sur l'exploitation de ce poisson sur le bassin méditerranéen (réalisation ISARA) ;

- un état des lieux de la population d'anguilles du Rhône débouchant sur des propositions d'actions (Université de Rennes). Dans le cadre de cette étude, une station de piégeage installée par le bureau d'études FISH-PASS en aval de l'aménagement de Vallabrègues, est suivie par l'association MRM. De plus, un transfert de civelles a été réalisé en novembre 1998 sur le cours médian du Rhône au niveau de la Réserve de l'lle de la Platière, dans les départements de l'Isère et de l'Ardèche, cette opération faisant actuellement l'objet d'un suivi par pêche électrique et par pêche aux engins pour tn mesurer les effets.

Dans le cadre du Plan Migrateurs Rhône-Méditerranée, les partenaires financiers de l'association Migrateurs Rhône-Méditerranée sont le Ministère de l'Aménagement đu Territoire et de l'Environnement, le Conseil Supérieur de la Pêche, l'Agence de l'Eau Rhône-Méditerranée-Corse, la Compagnie Nationale du Rhône, le Syndica: Intercommunal d'Aménagement du Bas-Gardon, le Syndicat Intercommunal Ardèche Claire, Électricité De France, Voies Navigables de France, l'Institution Interdépartementale du bassin Rhône-Saône, les Conseils Régionaux Languedoc-Roussillon, Provence-Alpes. Côte d'Azur et Rhône-Alpes, les Conseils Généraux de l'Ardèche, des Bouches du Rhôñ, du Gard et du Vaucluse.

\section{BIBLIOGRAPHIE}

ANONYME, 1999. Analyse stratégique du Plan Migrateurs Rhône-Méditerranée. BCEOM, NICAYA, Institut COHERENCE, 1999. Note de synthèse. 35 p.

GUILLARD J., COLON B., THERRY F., VIDAL N., 1998. La migration des aloses (Alosa fallax rhodanensis) dans l'écluse de Beaucaire-Vallabrègues étudiée par un sondeur acoustique à faisceaux partagés : synthèse des campagnes 1996 à 1998. INRA Thonon-les-Bains, Association MRM. Rapport I.L. 146-98. 45 p.

HOESTLAND H, 1958. Reproduction de l'alose atlantique (Alosa alosa L.) et transfert al Bassin méditerranéen. Verh. Internat. Ver. Limnol. 13: 736-742.

KIPPER Z.M., MILEIKOV I.V., 1968. Fishways in hydro developments in the USSR. Published for the US Department of the Interior and the National Science Foundation, Washington DC by the Israël Program for Scientific Translations. p. 42 46.

LEBEL I., BAGLINIERE J.L., PORCHER J.P., SABATIE R., 1999. Bilan des actions et dts études menées par l'Association Migrateurs Rhône-Méditerranée de 1991 à 1998 Association MRM, INRA Laboratoire Ecologie Aquatique de Rennes, CSP Délégation de Rennes. 57 p.

RAMEYE L., KIENER A., SPILLMANN C.P., BIOUSSE J., 1976. Aspects de la biologie d? l'alose du Rhône. Pêche et difficultés croissantes de migrations. Bull. Fr. Pềche Piscic.(1976) $263: 51-76$.

ROULE L., 1924. Les aloses des eaux douces de France. Bulletin de la Société Zoologiqư de France, 49, 265-266 